\title{
In-situ Electrokinetics Using Liquid Phase Transmission Electron Microscopy
}

\author{
Mads S. Larsen $^{1}$, Murat N. Yesibolati ${ }^{1}$ and Kristian S. Mølhave ${ }^{1}$
}

${ }^{1}$ DTU Nanolab, National Centre for Nano Fabrication and Characterization, Technical University of Denmark, DK-2800 Kgs. Lyngby, Denmark.

Observing in-situ nanoscale events using liquid phase transmission electron microscopy (LPTEM) [1] has opened for a wealth of new observations including nucleation of nanoparticles [2-3], dendrite growth [4], lithiation/delithiation studies [5] etc.

One of the challenges in LPTEM is however, that one of the major forces that exist on nanoscopic scale in liquids is the electrostatic force. The typical commercial LPTEM system is based on silicon nitride membranes (see Figure 1(a)), where surface charges are unavoidable, due to the interaction between silanol and silylamine sites to water [6]. Additional beam charging can also occur when illuminating the silicon nitride with the electron beam and radiolytic effects further complicate the reactive environment [7]. These charges can through electrokinetic effects have an effect on the flow inside nanoscale confined liquid [8].

We aim to quantify electrokinetic phenomena in LPTEM as they could be important given the many beam induced observations of slow or non-brownian motion of attached and freely moving particles [9-11]. Electrokinetic effects such as electroosmosis (EO) and electrophoresis (EP) may also open up for new control features of processes in LPTEM and understanding of beam induced effects, in addition for new characterization methods of nanostructures and processes.

We have shown it is possible to produce controlled electroosmotic flow, from which it becomes possible to deduce the zeta potential of the SiNx film as preliminary results from a STEM experiment, see Figure 1(c). The experiment was conducted using 300nL of a $1 \mathrm{mM} \mathrm{Na}_{2} \mathrm{SO}_{4}$ electrolyte in the clamped liquid cell.

Using a $20 \mathrm{MHz}$ Function Generator (AIM-TTi Instruments, TG2000), two different amplitudes of 6Vpp and $8 \mathrm{Vpp}$ were applied using a two-electrode configuration between the working electrode(s) (WE, ground, connected to a sensing electrode, $S$ ) and the counter electrode (CE connected to the reference electrode, RE) (Figure 1(b)). The 3 center electrodes were all connected to the WE to avoid any floating electrodes. The experiment was performed in a FEI Tecnai T20 at 200kV in STEM mode with a camera length of $100 \mathrm{~mm}$ and $14 \mathrm{kx}$ magnification, and recorded at $25 \mathrm{fps}$. Data was later analyzed using ImageJ (ver. 1.47t) in combination with Python (ver. 3.5).

The results clearly show that a difference in voltage amplitude corresponds to a higher EO pressure due to a increase in the gray level. The increase in EO pressure was also expected when comparing to an analytical model. The preliminary results shows how a thin, flexible, SiNx film can be investigated in terms the electroosmotic pressure, which in turn can reveal information of the surface charges and the corresponding zeta potential. The results can be further used when investigating EP flow of nanoparticles to deduce the contribution from EO flow, but also yield information about the contribution from the beam on the surface charges of the window, when compared to optical experiments conducted at 1 bar overpressure. 
In this way essential quantitative knowledge can be obtained for the surface charge state of samples in LPTEM.

\section{References:}

[1] F Ross in "Liquid Cell Electron Microscopy (Advances in Microscopy and Microanalysis)", (Cambridge University Press, Cambridge), p. 3.

[2] N Ahmad et al, Nano Letters 17 (2017), p. 4194.

[3] B Song et al, Nanoscale 10 (2018), p. 15809.

[4] Y Wu, and N. Liu, Chem 4 (2018), p. 438.

[5] M Gu et al, Nano Letters 13 (2013), p. 6106.

[6] SG Malghan, Colloids and Surfaces 62 (1992), p. 87.

[7] NM Schneider et al, Phys. Chem. C 118 (2014), p. 22373.

[8] F Balderassi, Journal of Colloid and Interface Science 325 (2008), p. 526.

[9] MN Yesibolati et al, Nano Lett. 20 (2020), p. 7108.

[10] TAJ Welling et al, Part. Part. Syst. Charact. 37 (2020).

[11] A Verch, M Pfaff and N de Jonge, Langmuir 31 (2015), p. 6956.

[12] We acknowledge financial support from DTU Nanolab at the Technical University of Denmark, Danish Research Council for Technology and Production Case No. 12-126194, and in part by the Advanced Materials for Energy-Water Systems (AMEWS) Center, an Energy Frontier Research Center funded by the U.S. Department of Energy, Office of Science, Basic Energy Sciences.

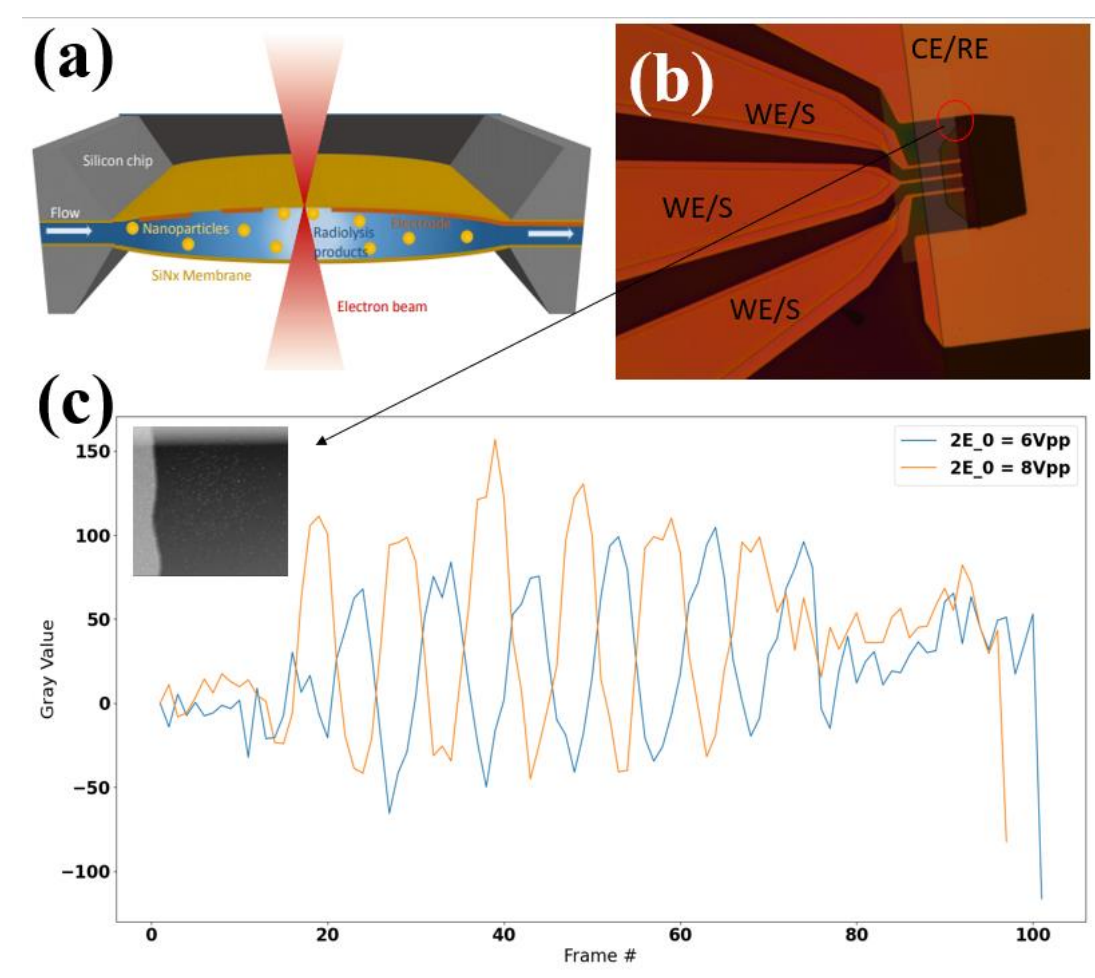

Figure 1. (a) Schematic of the LPEM cell (from [9], reprinted with permission from the author), (b) optical image of the top chip of the clamped system, electrodes are denoted as WE=Working electrode, $\mathrm{CE}=$ counter electrode, $\mathrm{S}=$ sensing electrode, and $\mathrm{RE}=$ Reference electrode, and (b) preliminary results using a $1 \mathrm{mM}$ sodium sulfate electrolyte; experimental details are explained in the text. 